\title{
SOBRE LOS FUNDAMENTOS FILOSÓFICOS DE LA DEMOCRACIA
}

\author{
Michelangelo Bovero
}

Departamento de Estudios Polfticos UNIVERSIDAD DE TURÍN

\section{Democracia ideal y democracia real}

Poco antes de la segunda guerra mundial, un fino estudioso de la historia de la cultura inglesa, Basil Willey, sugería el siguiente experimento: imaginemos que tenemos que explicar a un niño lo que significan términos como cristianismo, paz o democracia: "el método", afirmaba Willey, "si fuésemos bastante precisos, sería la sátira". En efecto, comentaba Willey, "la mejor sátira tiene como cbjetivo inducirnos a observar situaciones reales y conocidas como si fuese la primera vez", en consecuencia con los ojos de un niño "o como si fuésemos visitantes provenientes de un planeta como Utopía, de China, de Persia o de un imaginario cuartel general de la región”. Y explicaba que el inicio del siglo XVIII pudo presentar una situación particularmente favorable para el florecimiento de la época de oro de la sátira - la época de Dryden, Pope, Swift y Voltaire, y del Montesquieu de las Cartas persas - ciertamente por el predominio, en ese periodo, de la fe en la naturaleza y en la razón. De acuerdo con Willey, la verdadera sátira lleva a la "condena de la sociedad en referencia a un ideal", en cuanto ella consiste precisamente en "medir las aberraciones monstruosas con respecto al ideal". 1

Vale la pena leer en toda su extensión el fragmento en el que Willey describe la situación a principios de la época de oro de la sátira: "En aquel tiempo la oscuridad medievál había sido disipada suficientemente como para que los contornos de la situación moderna se volvieran claramente visibles. El hombre, considerado hasta entonces como un alma inmortal en busca de la salvación o un alma racional en busca de la virtud, ahora era visto como el

1 B. Willey, The Eighteenth Century Background. Studies on the Idea of Nature in the Thought of the Period, Chatto \& Windus, Londres, 1940, tr. it. en B. W., La cultura inglese del seicento e del settecento, Il Mulino, Bolonia, 1975, pp. 406, 402-3. 
ego político o económico en busca de riqueza, de poder y de una posición social. Durante el siglo XVII los ávidos apetitos todavía estaban escondidos tras las posiciones políticas y religiosas; pero ahora aparecía muy claramente que los individuos y las naciones aspiraban realmente a los bienes materiales del mundo, más disponibles que cuanto no lo hubiesen sịdo para los grandes conquistadores bárbaros o los hebreos de Malta. Sin embargo - y aquí está el meollo del asunto-, los viejos modelos sobrevivían como fantasmas, sentados con sus coronas sobre las tumbas del cristianismo y del humanismo; los hombres instintivamente se volvían hacia esos viejos modelos que, teóricamente, se suponía que todavía vivían. En consecuencia, cualquiera que todavía creyese firmemente en tales modelos, aun teniendo la capacidad de ubicar las cosas en su realidad, disponía de todos los elementos de la sátira: lo ideal y lo real en neta contraposición. ${ }^{2}$ ¿De nobis fabula narratur? (¿tenemos algo que ver con esta historia?) ¿Tal vez debamos buscar a través de consideraciones semejantes a las de Willey la razón del gran éxito que la sátira política, aunque ya no de manera escrita sino hablada y "diseñada", ha tenido entre nosotros?

Hace algunos años, con base en una observación sin ilusiones de los acontecimientos no exenta de un intento polémico, fue acuñada la expresión "democracia real" tomándola literalmente de la muy usual "socialismo real". ${ }^{3} \mathrm{Si}$ muchos fenómenos que se le atribuyen a la democracia real nos parecen aberrantes, por lo menos a algunos de nosotros, eso sucede justo porque tendemos implícitamente a comparar la realidad empírica con una imagen ideal de la democracia. Desafortunadamente se trata de una imagen más bien confusa, como confusos e inseguros son con frecuencia los juicios críticos o de condena de las aberraciones de la democracia real, como si esos juicios fuesen sólo producto de un malestar, de un sentido de descontento ético - por ello deben tratarse como manifestaciones de ingenuo moralismo- más que argumentos basados en una lúcida construcción racional. Lo que propongo es precisamente avanzar hacia la explicación de la comparación entre democracia ideal y democracia real que subyace en muchos de nuestros juicios políticos cotidianos. En el panorama de la reflexión política contemporánea encontramos dos tipos diferentes de contribuciones teóricas: existen, de una parte, algunos intentos de análisis conceptual de la democracia ideal, pocos y no siempre satisfactorios; de otra parte,

2 Jbid., p. 403.

- Cfr. Tra pace e guerra. Tra realismo dei blocchi e autodeterminazione dei popoli. Faccia a faccia tra Norberto Bobbio e Gian Giacomo Migone, a cargo de I. Stagliano, "Unita proletaria”, VIII, n. 1-2, 1982, pp. 77-84. 
hay una gran cantidad de análisis fenoménicos de las distintas formas y dimensiones de la democracia real. Lo que me parece poco desarrollado es precisamente el intento de vincular los dos tipos de análisis, es decir, de medir la distancia entre la democracia ideal y la democracia real, de investigar si, y en qué medida, la democracia real corresponde todavía a los principios de la democracia ideal, de descubrir dónde y por qué se produce el distanciamiento entre las dos, de comprender si tal distanciamiento es inevitable o corregible, de identificar mediante hipótesis racionales las condiciones bajo las cuales esta distancia podría reducirse. Moviéndonos en esta dirección, lo que ante todo se requiere es reconstruir el primer término de la comparación, o sea, el modelo ideal de democracia, y digo "ideal" no tanto en el sentido de meta deseable, sino en el sentido de concepto puro, de tipo ideal. ¿En qué otro lugar se deben buscar los fundamentos filosóficos de la democracia si no es en su concepto puro?

\section{La igualdad como categoría de la democracia}

Para sugerir este primer paso, me inspiré una vez más en Willey: imaginemos que debemos proporcionar a un extraterrestre -o a un persa (de los de Montesquieu, porque con un iraní de hoy el diálogo sería más difícil, y quizá involucraría otro tipo de recursos)-, los instrumentos conceptuales básicos para pensar la democracia. Ello equivale a preguntarse de la manera más directa e ingenua posible cuáles son las categorías fundamentales de la democracia; al mismo tiempo póngase atención en captar los eventuales pliegues satíricos, incluso involuntarios, de las respuestas. La respuesta que inmediatamente viene a la cabeza, antes de cualquier reflexión docta sobre el argumento, lleva a identificar los elementos indispensables para concebir una primera idea de democracia en alguna mixis (mixtura) de la igualdad y la libertad. Intentemos seguir la indicación que proviene de esta primera respuesta; pero propongo dejar entre paréntesis, por el momento, la libertad, porque ella se refiere más bien, en mi perspectiva, a lo que llamaré el principio de la democracia, distinguiéndolo de la verdadera y propia categoría que define la democracia con respecto a otras formas de gobierno: precisamente, la igualdad.

Lo que distingue a la democracia en casi todas las versiones que han sido presentadas de ella, en los tiempos antiguos o modernos, es alguna forma de igualdad, o mejor dicho de equilibrio, superación o absorción de desniveles. Inmediatamente nos viene a la memoria Tocqueville, quien ubicó la indeclinable tendencia de los modernos 
hacia la democracia en la erosión de la barrera entre lo alto y lo bajo de la sociedad y el Estado, hacia lo que él llamaba la "igualdad de condiciones" y hacia la igualdad de derechos políticos; pero los antiguos mostraban tener una idea semejante de su democracia - de la cual algunos historiadores de las cuestiones de la Antigüedad se obstinan en sostener la absoluta heterogeneidad con respecto a nuestra democracia, de acuerdo con muchos politólogos, que ven en la democracia moderna pocas semejanzas con la democracia antigua-, 4 considerándose sinónimo de democracia el término isonomía, literalmente "igualdad de ley" (es menos correcta la traducción común "igualdad frente a la ley"): de conformidad con la interpretación de Vernant ${ }^{5}$ la noción de isonomía propone una imagen de la democracia semejante a un círculo, en el que todos los puntos son equidistantes del "centro", sitio donde reside el poder, contrapuesta al modelo "piramidal", al que corresponde la imagen de las monarquías orientales. De isonomía como sinónimo de democracia - como tal se encuentra en Heródoto, no sólo y no tanto en los pasajes que sugieren a Vernant su interpretación geométrica, sino más claramente en el célebre pasaje donde se funda la teoría clásica de las formas de gobierno- ${ }^{6}$ se pueden dar interpretaciones ligeramente diferentes, como aquella propuesta recientemente por Moses I. Finley en

4 Sobre este punto G. Sartori, en el reciente The Theory of Democracy Revisited, Chatam House, Chatam (New Jersey), 1987, no hace más que refrendar la tesis sostenida desde Democrazia e definizioni, Il Mulino, Bolonia, 1957. En el término Democracy de la International Encyclopedia of the Social Sciences (Macmillan \& Free Press, Nueva York, 1968, vol. IV, ahora en tr. it. en el vol. Elementi di teorio politica, Il Mulino, Bolonia, 1987, que recoge diversos escritos de Sartori desde 1962 hasts la actualidad), después de haber afirmado que "mientras para los griegos la democracia literal era la única forma de democracia posible, para nosotros la democracia literal es una forma de gobierno imposible”, Sartori se pregunta "por qué hemos retomado - después de dos mil años de presencia y también de descrédito- un término cuyo significado original reclama una patente imposibilidad"; y piensa que el término haya mantenido sustancialmente el mismo significado, pero haya pasado del uso descriptivo antiguo a un uso sobre todo prescriptivo (cfr. op. ult. cit., p. 39).

5 Cfr. J. P. Vernant, Mito e pensiero presso i greci (1965), Einaudi, Turín, 1978, pp. 219-20. Aqui Vernant, apoyándose en Heródoto (III, 142, pero también IV, 161 y VII, 164), refiere la noción de isonomía a la ciudad-estado griega en general, cuyo "espacio político... simétricamente organizado alrededor de un centro, es construido de acuerdo con un esquema geométrico de relaciones reversibles, cuyo orden se basa en el equilibrio y la reciprocidad entre iguales" (ibid.); pero al ser representada de este modo y al ser contrapuesta a las monarquias, "la ciudad en general", decla el maestro de Vernant, Louis Gernet, "tiende a la democracia como si la democracia fuese el término necesario de su desarrollo": cfr. La nozione di democrazic tro i greci (1948), ahora en L. G. I greci senza mirocolo, Editore Riuniti, Roma, 1986, pp. 319-20.

Naturalmente me refiero al logos tripolitikos: Heródoto, III, 80-82. 
su última obra importante, La política en el mundo antiguo: para los atenienses "la palabra que nosotros traducimos como 'igualdad frente a la ley' significa igualdad a través de la ley, esto es, igualdad de derechos políticos de todos los ciudadanos, una igualdad que fue creada por una evolución constitucional, es decir, por la ley. Aquella igualdad no sólo significaba el derecho de votar, de ejercer los cargos públicos y así por el estilo, sino ante todo el derecho de participar en la elaboración de las directrices políticas en el Consejo y en la Asamblea". ${ }^{7}$

Poco más adelante Finley recuerda que a principios del siglo $\mathrm{V}$ se acuñó otro término que fue tomado como sinónimo de democracia, que también está presente en Heródoto, isegoría, que significa precisamente "libertad de palabra, no tanto con la sutileza negativa que la expresión ha tomado entre nosotros, en el sentido de libertad frente a la censura, sino en el sentido más denso de derecho de hablar en voz alta donde más importaba, en la asamblea de todos los ciudadanos" 8 Únicamente debe agregarse que el significado democrático de isegoría no radica en el ser una libertad sino, como resulta evidente por la construcción original del término, en ser una igualdad. Al respecto, vale la pena recuperar un curioso cuanto significativo pasaje, no citado por Finley, en el que aparece la palabra isegoría: en la Ciropedia de Jenofonte. Dirigiéndose al abuelo Astiages, rey de los medos, Ciro, menor de edad, cuenta haber asistido a una fiesta en la que el abuelo y sus amigos bebían vino abundantemente. "Vi - cuenta Ciro- que ustedes habían perdido el uso de la razón y de los miembros. Ante todo, no hay acción que ustedes nos prohiban a los niños, que ustedes mismos no hicieran: gritaron todos juntos como locos, tanto que uno no entendía las palabras del otro; verdaderamente cantaban de manera ridícula... Cada uno de ustedes exaltaba su propia fuerza, pero cuando se levantaban para bailar no sólo no lograban tomar el ritmo, sino que ni siquiera podían mantenerse en pie. Olvidaron completamente que tú eras el rey. Entonces fue cuando entendí verdaderamente lo que es la famosa isegoría." 9 Parece que Jenofonte, con intencional cambio de tiempo y lugar, desea satirizar aquí la isegoría democrática, presentándola como fruto de un estado de ebriedad, en el que se

7 M. I. Finley, Politics in the Ancient World, Cambridge University Press, Cambridge, 1983, tr. it. Laterza, Roma-Bari, 1985, p. 205.

8 Ibid., p. 206.

9 Jenofonte, Ciropedia, I, iii, 10, que cito en la tr. it. de C. Carena, Einaudi, Turín, 1962, p. 246. 
pierde el sentido de las distinciones y el respeto por la autoridad. ${ }^{10}$ In vino aequalitas (en el vino todos son iguales).

\section{Problemas de igualdad}

Pero ¿qué igualdad? Quizá es verdad, como sostenía Tocqueville, que las igualdades se atraen, y una jala a la otra. Tocqueville afirmaba: "no se puede concebir que los hombres sean absolutamente iguales en todo, excepto en un único punto. Terminarán, por consiguiente, en ser iguales en todo" $;^{11}$ y en estos términos no hacía más que recalcar, extremándola, una famosa afirmación de Aristóteles, de acuerdo con la cual "la democracia nació del hecho de que quienes son iguales en una cosa creen ser absolutamente iguales: en cuanto son igualmente libres, consideran ser iguales en todo". ${ }^{12}$ Así pues, es preciso distinguir una igualdad propiamente democrática, o sea, especificar qué forma o tipo de igualdad corresponde a la democracia como su "categoría". Esta necesidad de especificación surge porque "igualdad", como se sabe, es un concepto indeterminado, es un género que contiene infinidad de especies, y por ello tiene mil facetas. Ya que en sí misma la igualdad es simplemente una relación entre dos (o más) términos, tal relación debe ser determinada, o sea, especificada permanentemente, con base en la naturaleza de los términos (¿quiénes son los iguales?) y en los caracteres considerados como criterios en la construcción de la misma relación (¿en qué cosa son iguales?). De esta manera el problema 'igualdad' no es un problema unitario (más que desde un punto de vista lógico y matemático, desde el que se contempla la igualdad en abstracto como género): de suerte que no existe un problema de igualdad para quien se ocupe de cuestiones morales, sociales o políticas, sino muchos, tantos como son las igualdades. Por ello conviene determinar el problema, o mejor dicho especificar cuál es la dimensión de la igualdad pertinente para la democracia, respondiendo correctamente a las preguntas canónicas “iigualdad entre quién?” e “igualdad en qué cosa?”. ${ }^{13}$ Antes de intentar res-

10 Luigi Spina, en su útil trabajo sobre Il cittadino alla tribuna (Liguori, Nápoles, 1986), refiere esta ocurrencia de isegoría a un concepto "más privado que público", y por tanto le atribuye un significado más coherente con el origen del compuesto, es decir, el de "hablar a la par" (p. 42); pero la intención satírica antidemocrática me parece evidente.

11 A. de Tocqueville, La democrazia in America (1895-40), tr. it. a cargo de $N$. Matteucci en Scritti politici, Utet, Turín, vol. II, rist. 1981, pp. 73-74.

12 Política, V, 1.

13 El problema en general es planteado de la manera más clara por N. Bobbio, 8. v. Eguaglianza, en Enciclopedia del Novecento, vol. II, Instituto dell'Enciclopedia 
ponder, es oportuno detenerse todavía en una cuestión de carácter general. El juicio de igualdad -o sea el que se expresa con las proposiciones "A es igual a B" o bien "A y B son iguales" - tiene frecuentemente una estrecha vinculación con el problema de la universalidad, es decir, de la pertenencia de uno o más entes a una especie o a un género. Esta vinculación, y frecuente confusión, entre la igualdad y la universalidad se presenta de inmediato cuando nos ponemos ante la perspectiva de buscar si hay, y cuál es, la igualdad entre todos los hombres en cuanto tales, o sea, como ejemplares de la especie: bajo esta perspectiva en realidad no se busca otra cosa más que la definición de la identidad de la especie o del género, es decir, la definición de lo universal en la que todos los ejemplares se identifican. Propongo que lo mismo vale para todos los universales, en consecuencia para la igualdad entre los animales, o entre todos los ciudadanos, considerados como encarnaciones de la figura de ciudadanos, etc. Ahora bien, un juicio de igualdad entre dos o más entes (o incluso todos) considerados en cuanto miembros de un determinado género no indica una relación real entre estos individuos, sino solamente afirma una relación puramente ideal o mental. Considerando A o B como "hombres" o como "ciudadanos" (ambos ejemplares del género "hombre" o del género "ciudadano"), la relación que afirmamos subsiste entre $\mathbf{A}$ y $B$ es la igualdad de $\mathbf{A}$ y B pero - diría nuestro viejo amigo Hegel- la igualdad "en sí, o "para nosotros": no por esto la igualdad es también la relación que subsiste "para A y B" en toda circunstancia concreta. La relación de igualdad no describe la forma de la diferencia recíproca de A y B, su tratamiento de iguales o desiguales, o incluso el tratamiento igual o desigual que ellos pueden recibir en general o en circunstancias particulares de un tercer sujeto $\mathrm{C}$ (por ejemplo de las instituciones); esa igualdad es simplemente el resultado de una referencia nuestra de ambos a un mismo género. En otras palabras, la relación ideal de igualdad entre entes en cuanto pertenecientes a un mismo género no debe ser confundida con las relaciones reales de estos mismos entes, que también pueden ser relaciones de desigualdad. Se trata de dos planos distintos; el problema es ver si es posible instituir entre ellos una conexión adecuada. Considero que es posible una conexión, y que ella permite justificar o no justificar, bajo ciertas condiciones, igualdades y desigualdades reales.

¿Se puede decir que las eventuales relaciones concretas de desigualdad entre A y B sean todas moralmente equivocadas o injustas simplemente porque todos los hombres o los ciudadanos en cuanto

Italiana, Roma, 1977. Cfr. también G. Sartori, Elementi di teorio politica, cit., cap. IV, "Eguaglianza", pp. 87-100. 
tales, en consecuencia también A y B en cuanto hombres o en cuanto ciudadanos, son iguales? Seguramente no. En ciertos casos, sin embargo, igualdades y desigualdades reales entre dos (o más) sujetos se pueden considerar justas o injustas con base en la igualdad ideal de tales sujetos, o sea, en su asimilación a un mismo género universal. Tal posibilidad depende de dos factores: sobre todo depende de la manera en que es definido el género universal, o lo que es lo mismo, de la naturaleza de los requisitos de pertenencia al género; y ante todo depende de evaluar si tales requisitos son importantes para el problema del tratamiento igual o desigual de esos dos sujetos. Es obvio que no siempre la posesión equitativa de los requísitos de pertenencia al mismo género es suficientemente relevante para establecer un tratamiento igual de dos sujetos: aunque A y B son hombres, pueden no "merecer", por ejemplo, igual estima o consideración en innumerables circunstancias. Pero el punto más interesante es el que se refiere a la manera de definir lo universal, incluso porque parece tener implicaciones que tocan el controvertido problema de las relaciones entre juicios de hecho y juicios de valor. En efecto, el juicio de igualdad entre dos entes basado en la referencia de ambos a un mismo universal - al concepto de hombre, o de ciudadano, etc.- no es de por sí un juicio de valor; pero sabemos bien que el universal "hombre" puede ser definido de muchas maneras, incluso contrastantes: nos podemos limitar a una caracterización en términos puramente biológicos, o recurrir a términos como libertad, dignidad (humana), derechos (del hombre); lo mismo vale para el universal "ciudadano", etc. Ahora bien, si el concepto universal es definido mediante términos de valor, es decir, si a los caracteres que lo definen se atribuye explícita o implícitamente un valor, entonces, como consecuencia, se debe respetar o promover este valor en todos los miembros del género definido por tal concepto. Por tanto, si el concepto de hombre, o el de ciudadano, contienen en sí un valor, entonces del juicio de igualdad entre los hombres, o entre los ciudadanos (dos, algunos o todos), deriva la prescripción de tratarlos como iguales, o sea, de considerar y respetar en cada hombre o ciudadano el valor (o los valores) que lleva consigo al igual que cualquier otro hombre o ciudadano. De esto deriva también, inmediatamente, la injustificabilidad de desigualdades reales que sean imputables a un desconocimiento de tales valores, y la obligación moral de corregir tales desigualdades.

(En general se puede concluir que muchos problemas de desigualdad social y política pueden remitirse en última instancia a un problema de tratamiento igual o desigual de los individuos, justificado 
con base en un juicio que reconozca o no reconozca en ellos, de hecho, un valor igual.)

\section{La igualdad democrática y la libertad política}

Veamos ahora si estas consideraciones nos ayudan a responder mejor a nuestras preguntas iniciales: ¿qué igualdad corresponde propiamente a la democracia?; ¿igualdad entre quién?; ¿igualdad en qué cosa? De acuerdo con una idea común, la democracia es definida por la igualdad entre todos los ciudadanos, esencialmente en el derechopoder de participar en las decisiones colectivas. Tal vez en términos más rigurosos deberíamos decir que la constitución democrática es la que prescribe el tratamiento equitativo de todos los ciudadanos en lo que se refiere a la distribución de ese derecho-poder. Pero ¿con qué fundamento puede justificarse ese tratamiento? Como bien sabía Aristóteles, la verdadera cuestión es quién debe ser ciudadano, o mejor dicho: 1) cómo debe ser definido el concepto de ciudadano, es decir, con qué requisitos, y 2) qué extensión debe tener tal concepto, esto es, a cuáles y cuántos individuos ha de ser reconocida la identidad de ciudadano. Sabemos que la ciudad democrática antigua conoció muchas restricciones al respecto: en la mejor de las hipótesis, solamente los varones libres adultos residentes y autóctonos podían ser ciudadanos a pleno título; por su parte, la democracia moderna se caracteriza por la universalización de la ciudadanía política activa, o mejor dicho, por su extensión a todos los miembros adultos y autóctonos, o integrados, de la comunidad social. Por tanto, cayeron dos limitaciones, aquellas por las cuales el ciudadano debe ser varón y libre por nacimiento (o sea no esclavo). Pero lo que me interesa subrayar es que en uno y otro caso la justificación de la igualdad democrática, definida como igualdad de derechos políticos entre ciudadanos, radica en un mismo valor implícito, en la definición democrática de ciudadano (la definición o ideal de ciudadano que identifica la constitución democrática como tal): en efecto, la atribución a cada cabeza de un voto, sea en el caso del sufragio universal, sea en el caso del derecho político limitado a los varones libres, se basa en la presuposición de que todos los sujetos considerados son capaces de juzgar y deliberar en materia política, es decir, en lo que se refiere al interés común, y que en esta capacidad o dignidad politica no influyen eventuales diferencias de estamento social. Por lo que sería inicuo considerar tales diferencias económicas y sociales en general como relevantes para excluir a alguien del derecho-poder de intervenir en el proceso político, o sea, para establecer desigualdades políticas. 
Ésta no es sólo la cláusula fundamental de la democracia (ideal) moderna: es el fundamento o presupuesto indispensable de la democracia en su concepto, incluida la de los antiguos. La doctrina de Protágoras, contenida en el diálogo platónico homónimo, expresa esta convicción a través del mito de la distribución a todos los hombres, por parte de Zeus, de la politiké techne, el arte del juicio político, donde se considera justo que el parecer individual en materia política deba ser escuchado como el de cualquier otro. ${ }^{14}$ En el mismo presupuesto de la correspondiente dignidad política está basada la igualdad en la elegibilidad de los cargos públicos, lo que los antiguos llamaban isotimia, y que corresponde al principio democrático moderno de la accesibilidad de todos, sin obstáculos económicos y sociales, a las funciones de mando. ${ }^{15}$ En la célebre apología de la democracia que Tucídides atribuye a Pericles se dice que cualquiera que sea capaz de ocuparse de sus intereses personales también es capaz de ocuparse de política, o sea, del interés público que es el interés de todos; por lo que quien no se ocupa de política no puede ser considerado un hombre tranquilo, sino más bien un inepto. ${ }^{16}$ Así pues, entre la democracia de los antiguios y la de los modernos - por lo menos siguiendo ciertas versiones ideales de ambas - no ha cambiado sustancialmente el concepto de ciudadano, que se identifica con el individuo considerado como sujeto capaz de voluntad racional, por ello mismo dotado de dignidad política; lo que ha cambiado sustancialmente es la concepción antropológica con base en la cual era reconocido como un sujeto capaz y digno sólo el varón libre por nacimiento.

\section{Platón, Protágoras, 322c-323b.}

15 Pero debe subrayarse que, mientras que en las democracias reales modernas estas barreras, abolidas de derecho, operan de hecho fuertemente, en la democracia real ateniense tenían al parecer una incidencia mucho menor, probablemente debido al mecanismo institucional diferente de selección de los jefes. Como dice Gernet (op. cit., p. 320), los griegos "aman mucho 'mandar" Mas debido a que no es posible que todos manden al mismo tiempo, se alternan en el mando. $Y$ ya que todos son igualmente competentes, se debe disponer de un medio que asegure la alternancia. Este medio es la extracción por sorteo, procedimiento venerable ahora despojado de su antigua virtud, que era de indole religiosa. Finley afirma que "desde el nacimiento cada niño ateniense teńa algo más que la probabilidad puramente hipotética de convertirse en presidente de la Asamblea, un cargo basado en un sistema de alternancia, que era asignado por un solo día y, como de costumbre, por sorteo. Además aquel mismo niño podía volverse comisario del mercado por un año, miembro del Consejo durante uno o dos años (con tal de que no fuesen consecutivos), ocupar repetitivamente un juzgado $y$, en fin, participar en la Asamblea con derecho a voto todas las veces que lo deseaba" (La democrazia degli antichi e dei moderni, Laterza, Roma-Bari, 1973, p. 20).

16 Cfr. Tucídides, La guerra del Peloponeso, II, 37-40. 
El análisis de la igualdad como categoría de la democracia nos ha llevado de esta manera, a través del problema de la justificación de tal igualdad democrática, a identificar el principio de la democracia. Si una cierta igualdad define la democracia en su especificidad entre las formas de gobierno - y podríamos concluir en este punto con las palabras de Aristóteles: "La democracia se define en primer lugar como el régimen en el que rige la igualdad: la ley de la democracia entendida de esta manera establece como norma de igualdad que los pobres no deban tener menos poder que los ricos, ni unos deban ser más que los otros amos del gobierno"_- ${ }^{17}$ el principio, el fundamento en el sentido de presupuesto y punto de partida ineludible de la democracia, es el individuo sujeto de voluntad racional, considerado abstractamente con respecto a sus condiciones económicas y sociales. Lo mismo en la democracia moderna que en la de los antiguos: simplemente no todos aquellos que son considerados individuos racionales por los modernos eran tales para los antiguos. Digo principio como punto de partida fundamental porque, si el problema político esencial, el problema de cualquier forma de gobierno, es el de llegar para toda cuestión de relevancia pública a una decisión colectiva unívoca, o sea, a una voluntad única, que deba considerarse como voluntad general o colectiva, superando el conflicto, el contraste o la simple heterogeneidad de las muchas voluntades de los coasociados, para la democracia (ideal) se trata de reducir las muchas voluntades individuales a una única voluntad colectiva de manera que, no sólo las voluntades individuales reconozcan en la colectiva una voluntad no externa, no impuesta, sino que la voluntad colectiva o general pueda efectivamente ser remitida a las individuales como a las fuentes originales de las cuales sólo ella puede brotar. En democracia, o por lo menos en la pureza ideal de su concepto, parece que todo ciudadano debe poder reconocer como propia la voluntad general, en cuanto ha contribuido a su formación: en este sentido al individuo racional como ciudadano activo, en cuanto principio, de la democracia, le ha sido reconocida la dote exclusiva de la libertad como autonomía, llamada también libertad positiva o política... jo libertad de los antiguos!

\section{Democracia de los antiguos y de los modernos}

Al llegar a este punto conviene una digresión para enfrentar la clásica duda, la libertad política como autonomía, ¿quizá no es solamente de los antiguos?; ¿tal vez no es verdad que se refiera a la democracia antigua en cuanto democracia directa, y por ello resulta 
excluida a priori de la democracia moderna en cuanto democracia representativa? Esto equivale a preguntarse si la democracia moderna es todavía democracia, o si lo es en un sentido completamente diferente e incomparable con el antiguo. Hasta ahora en nuestro breve recorrido había aparecido la duda contraria, también ampliamente difundida, de que fuese la democracia de los antiguos la que no era plenamente democracia, a causa de su restringida base popular. Aquí sostendré la tesis de que la democracia es una en su concepto, que tal concepto es definido por lo que he llamado fundamentos filosóficos de la democracia, o sea, por una cierta categoría de igualdad y un cierto principio individual, y que así la democracia moderna como la antigua, en la especificidad de las respectivas instituciones, son democracias solamente en la medida en la que puedan remitirse a esos fundamentos conceptuales.

Todo se reduce a la cuestión de si la diferencia entre la democracia directa de los antiguos y la democracia representativa de los modernos es una diferencia esencial, es decir, una diferencia en los fundamentos. Ante todo, es preciso regresar al primero de los caracteres esenciales o fundamentales del concepto de democracia en los términos en los que ha sido definido aquí, la igualdad entre todos los ciudadanos en el derecho-poder de participar en las decisiones colectivas, y evaluar si se adapta bien a ambas (pretendidas) formas de democracia. Contemplando la primera dimensión de tal igualdad, o sea, a los sujetos entre los cuales ella dèbe valer, se puede sostener que la democracia antigua no es propiamente tal porque excluye del ámbito de los "iguales" a un gran número de individuos, principalmente a los esclavos y a las mujeres. Se trata de una exclusión altamente relevante desde el punto de vista de los modernos; pero ella se refiere más bien a las concepciones antropológicas de los antiguos que a sus concepciones políticas. En la perspectiva antropológica de los antiguos, los esclavos y las mujeres entran en el ámbito "por naturaleza" inigualitario del poder doméstico, no en el ámbito del poder político: por este motivo son excluidos de cualquier forma política. De acuerdo con Aristóteles, para ellos "no hay polis". En consecuencia, su "inigualdad" con respecto a los varones libres no incide en su definición y distinción de las diferentes formas de la relación política, es decir, de la relación entre gobernantes y gobernados; pero es precisamente en el contexto de la distinción o clasificación de las formas políticas que la democracia es definida como el régimen igualitario por excelencia, en cuanto considera irrelevantes las diferencias económico-sociales para los fines de la distribución de los derechos políticos entre los miembros de la ciudad. En tratar a los pobres igual que a los ricos, recono- 
ciendo a los primeros como sujetos políticos activos tanto como a los segundos, radica el primer fundamento de la democracia, un fundamento puesto por los antiguos; incluso se puede decir que solamente la atribución, en muchos casos tardía, del derecho al voto sin distinción de censo (patrimonio) ha adecuado la democracia moderna al espíritu (concepto) de la democracia antigua.

Si de la primera dimensión de la igualdad democrática, "quiénes son los iguales", pasamos a considerar la segunda dimensión, "en qué cosa son iguales" - y sabemos que los ciudadanos deben serlo en el derecho-poder de participar en las decisiones colectivas-, la democracia de los modernos es la que no parece merecer plenamente el propio nombre, en cuanto los ciudadanos modernos no participan en las decisiones políticas más que eligiendo representantes que deciden en su lugar. En pocas palabras, la moderna no sería propiamente democracia porque es representativa y electiva. Aristóteles sabía bien que la elección de por sí, en su concepto, no es un procedimiento democrático, sino aristocrático: ${ }^{18}$ es una selección, y no se justifica más que como selección "de los mejores", de un hombre o de un partido como mejor que otro. Si literalmente fuese verdad que nosotros los modernos resolvemos la esencia de la democracia en el procedimiento de elegir a los gobernantes, deberíamos admitir haber cometido un error conceptual. No hay duda de que el eje del sistema que llamamos democrático es la elección; pero es necesario agregar: no la elección pura y simple, sino la repetición de la elección, que contiene en sí la posibilidad de reelección o de revocación. Y la repetición hace en principio (nos estamos moviendo siempre en el terreno de lo ideal, no de lo real) democrática a la aristocracia o a la oligarquía electivas. Mientras la simple elección de los gobernantes, esto es, la designación en las funciones decisivas, es una forma de juicio sobre hombres (o partidos), acerca de quién sea el mejor (aristos) o el más apto para decidir, y en este sentido, con respecto a la sustancia de los problemas colectivos, es una no-decisión, la elección sistemáticamente repetida es una forma de enjuiciar las decisiones, los resultados de las decisiones ya tomadas y los programas para las decisiones que deben ser tomadas. Así pues, a su manera, es una decisión en referencia a los problemas colectivos. Michel Walzer sostuvo recientemente que: "Es un signo característico de un gobierno democrático que las experiencias del líder no sean extrañas a los ciudadanos comunes. Con un pequeño esfuerzo de imaginación el ciudadano puede ponerse en el lugar de su representante elegido. Desde el momento en que lo puede hacer y, más aún, desde el momento en que normalmente lo hace, él toma 
parte en lo que me gusta definir... como un proceso de decisión que anticipa y que es retrospectivo... Este proceso de decisión vicario precede y sigue al efectivo proceso de decisión".$^{19}$ Pero lo que importa es que este proceso de decisión "vicario" culmina en el momento de la elección en una decisión efectiva, incluso en la decisión que orienta el curso futuro de decisión. En este sentido, se puede decir que en la democracia representativa todos los ciudadanos pueden participar en el proceso de decisión, al tener en el derecho de votar el poder de determinar el curso de tal proceso. En referencia a la democracia directa, lo que cambia no es tanto la igualdad en el derecho de participar en las decisiones, sino la estructura del proceso de decisión.

\section{El proceso democrático y sus peligros}

Sin embargo, precisamente la diferencia en el proceso de decisión modifica de manera significativa la fisonomía del sistema: la figura del círculo, acertadamente sugerida por Vernant basándose en Heródoto para la isonomía antigua, ya no es una representación adecuada para la democracia de los modernos. Efectivamente el poder de decisión de la base permanece igualmente distribuido entre los ciudadanos, pero el poder de decisión última se separa del nivel de la base: el poder ya no está "en el centro", o sea, en el mismo plano que los ciudadanos, al alcance de todos y equidistante de cada cual, sino que se trasladó a lo alto, a un "vértice". Con esto, el sistema se asemeja más bien a una pirámide, esto es, a la figura que según Vernant representa correctamente, en contraste con la isonomía griega, la autocracia oriental. Así y todo, si la pirámide - continuando con el juego de las figuras geométricas- répresenta un proceso de decisión político en varios grados, tal prodeso puede ser recorrido en dos sentidos: de arriba hacia abajo, o dé abajo hacia arriba. La autocracia se identifica con el proceso descendente: el principio está en el vértice, en el poder del autócrata que se impone, y que mediante un sistema de investiduras camina de lo alto hacia la base, es decir, al nivel de los súbditos privados de cualquier poder y derecho; la democracia representativa moderna se identifica con el proceso ascendente: el principio está en la base, en las muchas voluntades de los individuos concebidos como sujetos racionales autónomos, y mediante un sistema de nóminas camina de abajo al vértice, o sea, hasta los órganos habilitados para tomar decisiones colectivas

19 M. Walzer, Political Decirion-Making and Political Education, en M. Richter (ed.), Political Theory and Political Education, Princeton, 1980: tomo la cita de M. I. Finley, La politica nel mondo antico, cit., p. 44. 
con base en la suma de las decisiones individuales, manifestadas (principalmente) en el momento electoral. La vinculación entre las decisiones iniciales de los individuos y las decisiones colectivas que finalmente toman los elegidos está garantizada por la repetición frecuente de las elecciones, que implica la posibilidad de destitución. En este sentido, el individuo, concebido como sujeto de voluntad racional y por ello dotado de dignidad política, permanece también como el principio de la democracia representativa de los modernos: si el proceso que desde el principio conduce al resultado político final no es alterado, también el individuo moderno como ciudadano activo contribuye a la formación de la voluntad general y, en la medida de esta contribución, es el heredero legítimo de la "libertad de los antiguos".

Pero la representación "piramidal" del proceso de decisión ascendente pone en evidencia otras características de la democracia moderna, altamente relevantes en la perspectiva de nuestro discurso. En primer lugar, los múltiples planos intermedios que se insertan entre la base y el vértice son ocupados por organizaciones formales e informales (partidos, sindicatos, grupos de presión, etc.) cuyos miembros son, con respecto al ciudadano común, "más cercanos" al momento culminante de la decisión política, y por tanto tienen la oportunidad de influir más en su contenido. Es como decir: todos los ciudadanos son iguales, pero unos son más iguales que otros. En segundo lugar, y consecuentemente, la orientación de la base, definida por las decisiones iniciales de los ciudadanos electores, puede ser desviada y distorsionada al subir los diferentes niveles, y todo el curso de decisión puede cambiar de dirección, cuando las organizaciones intermedias adquieren fuerza y se convierten en lugares de poder independiente, más o menos discrecional. En tal caso, el proceso de decisión política permanece como un proceso ascendente, pero ya no sigue estrictamente la dirección indicada por los ciudadanos, llegando a resultados finales más o menos distantes de las intenciones de estos ciudadanos. De esta manera, las expectativas manifestadas por los electores en la adhesión a un determinado programa político, pueden ser continuamente frustradas. Es evidente que en este caso los individuos no pueden reconocer como propia la voluntad formulada en las decisiones tomadas en el vértice.

En tercer lugar, el poder discrecional que los organismos ubicados en los niveles superiores de la pirámide política adquieren con facilidad, puede cambiar radicalmente el curso ascendente del proceso de decisión, modificando con esto su carácter democrático y transformándolo en un proceso autocrático. El mayor poder discrecional que los organismos políticos verticales (institucionales y 
no institucionales) ejercen normalmente se puede considerar como una extensión del llamado "poder de agenda" (poder de definir la orden del día): es el poder de presentar a la opinión pública, y en consecuencia a la decisión de los ciudadanos electores, ciertos problemas y no otros, de formular los asuntos políticos en ciertos términos y no en otros, de indicar ciertos criterios de solución y no otros. Cuando tal poder queda en manos de organismos o grupos restringidos que lo ejercen sin un control efectivo de parte de la opinión pública, o sea, de parte del conjunto de los ciudadanos que operan ese "proceso de decisión vicario" descrito por Walzer, cuando es obstaculizada para la mayor parte de los ciudadanos la posibilidad de crítica y de intervención eficaz en la formulación de propuestas, programas y orientaciones políticas, entonces el individuo ya no es el principio del proceso de decisión; este proceso tiene en realidad un punto de partida diferente. y presenta por lo menos un primer signo descendente, esto es, autocrático. Tal segmento descendente, que se mueve del planteamiento vertical de los problemas políticos a las selecciones electorales de la base, limita la libertad de decisión de los ciudadanos, o incluso puede llegar a manipular su capacidad de selección. Aunque luego el proceso político sube de la base al vértice, de las decisiones de los ciudadanos electores a las decisiones colectivas finales, el juego democrático ahora aparece falseado.

El carácter autocrático de este segmento descendente del proceso de decisión se manifiesta en el uso astuto de los medios de comunicación, que a veces muestra una tendencia hacia un verdadero y propio monopolio del poder ideológico, es decir, del poder de control sobre las conciencias. El poder de grupos restringidos pretende imponerse mediante un disfraz de democracia, tratando de engañar a los ciudadanos, a los cuales finge reconocer dignidad y capacidad de juicio político, presentando problemas en forma distorsionada, proporcionando criterios de juicio manipulados. En estas condiciones, la elección cae en el riesgo de volverse un puro rito de legitimación exterior, y el proceso político democrático en parte es sustituido por procesos de decisión inaccesibles para la mayor parte de los ciudadanos, o incluso invisibles y ocultos (los poderes secretos), en parte es acompañado por procesos no democráticos en cuanto no pueden remitirse a los fundamentos de la democracia (considero que entran en esta categoría los llamados procesos neocorporativos). En la medida en que estas tendencias logran prevalecer, la democracia real se divorcia de la democracia ideal y se transforma en una democracia aparente, en una máscara engañosa. 


\section{Democracia y modernidad}

Sin embargo, para no dejarse arrastrar por un pesimismo excesivo y no ser inducidos a creer que la democracia sea solamente un fantasma irreal, quizá sea necesario reflexionar sobre algunas preguntas: ¿por qué el poder vigente en nuestros sistemas políticos reales, incluso en los más criticables, busca de alguna manera una legitimación mediante los procedimientos de la democracia ideal?; ¿por qué casi no existe sistema autocrático o totalitario que no intente presentarse como democrático o justificarse como necesario para preparar la llegada de la democracia?; ¿por qué, en suma, la única forma de poder legítimo, por consenso universal incluso de los autócratas, es en nuestro tiempo la democracia? En el tiempo de los antiguos no era así: aristocracia y monarquía eran consideradas formas legítimas de gobierno, alternativas a la democracia. Más aún: eran juzgadas por la mayor parte de los doctos como mejores que la democracia. El hecho es que modernidad y democracia están entrelazadas en un nexo complicado, contradictorio pero indisoluble. El principio de la modernidad reside en un concepto universal del valor del hombre en cuanto tal: un concepto desconocido para los antiguos, que encuentra su correcta expresión solamente en las declaraciones modernas de los derechos del hombre. Es el principio de la libertad subjetiva, como lo definía Hegel, con base en el cual cada individuo reivindica el derecho de juzgar y de decidir por sí mismo, de definir sus creencias y de autodeterminarse con base en ellas: es el derecho de no vivir como objeto actuado sino como sujeto actuante, capaz de pensar y tener una voluntad propia. En el plano de los principios, la dimensión política de la modernidad no puede ser más que la democracia. El proceso que ha llevado a la condición actual, en el que todos los sistemas políticos son más o menos distantes de la democracia ideal, pero en el que ninguno de esos sistemas puede ser considerado legítimo si no es democrático, testifica al mismo tiempo la vitalidad y la fragilidad del principio moderno. Ciertamente el análisis teórico y la experiencia histórica muestra la dificultad intrínseca de su realización completa. La democracia es difícil, quizá es imposible, pero, paradójicamente, es al mismo tiempo inevitable: la democracia puede ser deformada o también falseada en algunos regímenes, puede ser cambiada y reprimida en otros, pero no puede ser marginada o suprimida. Por lo menos en tanto que creamos en el principio moderno de la dignidad del hombre. 